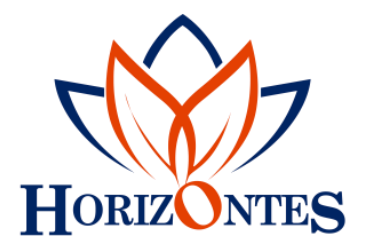

www.revistahorizontes.org

\title{
Teorías científicas. Las propuestas de Popper y Kuhn sobre investigaciones científicas
}

\author{
Scientific theories. Popper and Kuhn's proposals on scientific research
}

Teorias científicas. As propostas de Popper e Kuhn sobre pesquisa científica

ARTICULO DEREVISIÓN

\author{
Jimy Humpiri Nuñez \\ jepistemologia@gmail.com \\ ORCID: 0000-0003-0655-8403
}

\author{
Flor de Maria Humpri Nuñez \\ florxhiita@gmail.com \\ ORCID: 0000-0001-5533-3433
}

\author{
Eddy Erik Mamani Condori \\ eryk.ded@gmail.com \\ ORCID: 0000-0002-4433-8957
}

\section{Universidad Nacional del Altiplano UNA-PUNO, Perú}

Recibido 15 de febrero 2021 | Arbitrado y aceptado 05 de marzo 2021 | Publicado en marzo 2021

\section{RESUMEN}

Esta investigación tiene como objetivo analizar las teorías científicas según los postulados de Popper y Kuhn sobre investigaciones científicas. La metodología empleada es un diseño bibliográfico de tipo documental. Como conclusión, el proceso de formulación de teoría en el desarrollo de la ciencia, condicionadas a su contrastación, refutación y nueva conjetura constante en búsqueda de una mayor certeza en el conocimiento científico según la postura de Popper, la misma que podría ser condicionado no solo a la postura de Kuhn en las revoluciones científicas y su inconmensurabilidad terminológica, condicionado al desarrollo de la naturaleza y la sociedad. Es por ello, que dentro del desarrollo de la ciencia sujeta a la conjetura y refutación, ensayo - error y el proceso de falsación y contrastación, queda abierta la discusión de la inconmensurabilidad, no solo en la transmisión del lenguaje, sino, la concepción del cambio inevitable tanto del tiempo-espacio-fenómenoinvestigador; como dentro conocimiento científico.

Palabras clave: Ciencia; contrastación; falsación; paradigma, inconmensurabilidad

\begin{abstract}
This research aims to analyze scientific theories according to the postulates of Popper and Kuhn on scientific research. The methodology used is a documentary-type bibliographic design. As a conclusion, the theory formulation process in the development of science, conditioned to its contrast, refutation and constant new conjecture in search of greater certainty in scientific knowledge according to Popper's position, which could be conditioned not only to Kuhn's position in scientific revolutions and its terminological incommensurability, conditioned to the development of nature and society. That is why, within the development of science subject to conjecture and refutation, trial - error and the process of falsification and contrast, the discussion of incommensurability remains open, not only in the transmission of language, but also the conception of language. Inevitable change both of time-space-phenomenon-researcher; as within scientific knowledge.
\end{abstract}

Key words: Science; contrast; falsification; paradigm, incommensurability 


\section{RESUMO}

Esta pesquisa tem como objetivo analisar teorias científicas a partir dos postulados de Popper e Kuhn sobre a pesquisa científica. A metodologia utilizada é o desenho bibliográfico do tipo documental. Conclui-se o processo de formulação de teorias no desenvolvimento da ciência, condicionado ao seu contraste, refutação e constantes novas conjecturas em busca de maior certeza no conhecimento científico segundo a posição de Popper, que poderia estar condicionada não apenas à posição de Kuhn nas revoluções científicas e sua incomensurabilidade terminológica, condicionada ao desenvolvimento da natureza e da sociedade. É por isso que, no desenvolvimento da ciência sujeita a conjecturas e refutação, tentativa - erro e processo de falsificação e contraste, a discussão da incomensurabilidade permanece aberta, não apenas na transmissão da linguagem, mas também na concepção da linguagem. Mudança inevitável ambos de pesquisador de fenômeno-espaçotempo; como dentro do conhecimento científico.

Palavras-chave: Ciência; contraste; falsificação; paradigma, incomensurabilidade

\section{INTRODUCCCIÓN}

Existen numerosas teorías científicas las cuales han surgido gracias a la persecución de los investigadores donde han comprobado ciertos postulados que se han transformado en leyes elementales de nuestro mundo. Con este protocolo nace la ciencia. En otras palabras, la ciencia está formada por conocimiento ordenados cuya veracidad se puntualiza constantemente en el curso de la práctica social, hecho que le convierte en una disciplina que utiliza el método científico con la finalidad de hallar estructuras generales o leyes (Palella, y Martins, 2010).

El método científico se transforma en la parte fundamental del desarrollo de la ciencia. Es un método que integra la inducción y la deducción, con la finalidad de construir conocimiento teórico y aplicado a través de planteamiento del problema, formulación de posibles hipótesis, puestas a prueba de las hipótesis y resultados y derivación de las consecuencias (Sánchez, Reyes, y Mejía, 2018).

Para construir el conocimiento es necesario aplicar la investigación en todo su esplendor. La investigación se realiza a través de pasos que permiten conseguir los problemas más urgentes que pueda tener la sociedad. Es entonces un proceso de recolección de datos para dar respuestas a las preguntas o interrogantes planteadas, referidas al conocimiento de una realidad o a su transformación, la cual puede ser básica o aplicada, sustantiva o tecnológica (Sánchez, Reyes, y Mejía, 2018). Del mismo modo, la investigación es un conjunto estructurado de procedimientos teóricos, metodológicos y técnicos que se emplean para conocer e interpretar los aspectos esenciales, las relaciones fundamentales de un determinado objeto o fenómeno de la realidad (Garcés, 2000).

De manera general la investigación puede tener dos orígenes, uno empírico creado por el saber popular. Es decir, es la forma común, corriente y espontánea de conocer, que se adquiere con el trato directo entre los seres humanos y los objetos, por lo cual es subjetiva, asistemática y acrítica (Méndez, et. al., 2001).

El otro tipo de investigación es la científica las cuales ejecutado por expertos en el área. Se refiere a una secuencia lógica de actividades que procura descubrir las características de los fenómenos, las relaciones internas entre sus elementos y sus conexiones con otros fenómenos, mediante el raciocinio y la comprobación a través de la demostración y la verificación (Méndez, et.al., 2001). La investigación científica se encarga de producir conocimiento (Morone, 2013). Se caracteriza por: 
Que sea sistemático significa que no puedo arbitrariamente eliminar pasos, sino que rigurosamente debo seguirlos.

Que sea metódico implica que se debe elegir un camino (método: camino hacia), sea, en este caso, una encuesta, una entrevista o una observación.

Que sea racional / reflexivo implica una reflexión por parte del investigador y tiene que ver con una ruptura con el sentido común. Hay que alejarse de la realidad construida por uno mismo, alejarse de las nociones, del saber inmediato. Esto permite llegar a la objetividad. Que sea crítico se refiere a que intenta producir conocimiento, aunque esto pueda jugar en contra. (Morone, 2013, p. 1).

Como la investigación es un acto protocolar la cual tiene énfasis en etapas y actividades, por lo que cada una de ellas es fundamental para la consecución del objetivo general del mismo. Los objetivos de una investigación son una construcción teórica porque define: primero, qué se habrá de investigar; segundo, las perspectivas desde las cuales se lo hará; y tercero, la metodología apropiada para esa teoría y esos objetivos (Sautu, 1997).

En este sentido, el investigador juega un papel clave para el desarrollo de la investigación y por ende del conocimiento que desea encontrar. Este actor tiene además el rol de generación de conocimiento sobre el problema en sí, integrándolo con el conocimiento que sobre él tenga la población afectada $y$ otros actores relacionados (Alzugaray, Mederos, y Sutz, 2011). Por lo cual, un buen investigador debe cumplir ciertas características tal como se detalla en la Tabla 1.

Tabla 1. Deberes de un buen investigador.

Un buen investigador identifica claramente lo que recoge de otros autores, y da crédito incluso a las buenas ideas que ha recibido verbalmente de otros.

Un buen investigador domina adecuadamente los sistemas de citación y de elaboración de notas.

Un buen investigador está al día en su propia disciplina: conoce a los principales autores y conoce la discusión relevante. De esta manera evita presentar erróneamente, como novedosas y originales, teorías que ya han sido formuladas por otros, y, de paso, disipa toda sospecha de plagio.

Un buen investigador conoce la ley sobre propiedad intelectual que rige en su país. Puesto que esta ley es relevante para su trabajo y se presume justa si procede de autoridad legítima (que es lo normal), el investigador debe conocerla a lo menos en general. Con todo, puesto que las prohibiciones que se establecen en este tipo de leyes no son siempre absolutas desde el punto de vista moral, ciertas circunstancias pueden eximir de su cumplimiento. 
Un buen investigador, cuando envía a publicación una obra substancialmente idéntica a otra que ha publicado previamente, siempre expresa esta circunstancia. Desde luego al editor, pero también a los potenciales lectores. Y siempre en un lugar de fácil acceso.

En su lista de publicaciones (por ejemplo, en un curriculum vitae), un buen investigador identifica claramente las reimpresiones o nuevas versiones de una publicación previa.

Si la nueva es substancialmente igual a la anterior, el buen investigador la agrega dentro del mismo número, para evitar que una publicación en duplicado cuente como si fueran dos trabajos diversos.

Un buen académico investigador no deja sin sanción el plagio que descubre en sus alumnos. La condescendencia con el plagio solo cauteriza la conciencia del plagiario y torna más difícil el combate contra esta práctica que, como se indicó anteriormente, pone en serio riesgo el prestigio de la labor científica y de la institución universitaria como el lugar propio de ella.

Fuente: (Miranda, 2013)

El investigador también tiene un papel a jugar en la generación de los mecanismos que permitan la integración efectiva de todos los actores del sistema cuya participación es necesaria para la solución efectiva del problema (Alzugaray, Mederos, y Sutz, 2011). Es por ello, que la creación de redes de información puede ayudar a seguir fomentando el conocimiento entre los especialistas. Estas redes comunican los avances en la investigación, así como de entrelazar colaboraciones conjuntas en pro del desarrollo científico. En definitiva, el propósito de las redes es mantener actualizados a la comunidad, en los últimos avances del conocimiento, con el fin de impulsar la investigación básica y aplicada de punta, que permitan la consolidación de comunidades científicas alrededor del mundo (Romero, 2002).

Sin duda alguna, el protagonista de toda esta convergencia de personas y situaciones es el conocimiento que se esta formando. Por lo cual, el conocimiento puede ser entendido de diversas formas: como una contemplación porque conocer es ver; como una asimilación porque es nutrirse y como una creación porque conocer es engendrar (Martínez y Ríos, 2006). De la misma manera, se refiere a la información adquirida por una persona a través de la experiencia o la educación, así como también se entiende como la comprensión teórica o práctica de un asunto referente a la realidad (Sánchez, Reyes, y Mejía, 2018).

Una parte importante en la generación de conocimiento es la información, por lo que es fundamental para tal fin. Para transformarse en conocimiento, la información debe ser evaluada como verdadera o falsa, pertinente o irrelevante, práctica o impráctica, interesante o tediosa, además esta puede ser pública o privada, mientras que el conocimiento es personal (Bunge, 2003). En otras palabras, la información más la evaluación es el conocimiento generado. Si este conocimiento es científico entonces debe tener ciertas características, según Bunge (1965):

- Es fáctico. Parte de los hechos, los respeta hasta cierto punto $\mathrm{y}$ siempre vuelve a ellos.

- Trasciende los hechos. Va más allá de los hechos, produce nuevos hechos y los explica. 
- Es claro y preciso. Sus problemas son distintos, sus resultados son claros.

- Es comunicable. No es inefable sino expresable, no es privado sino público.

- Es verificable. Debe aprobar el examen de la experiencia.

- Es sistemático. Una ciencia no es un agregado de informaciones inconexas sino un sistema de ideas conectadas lógicamente entre sí.

- Es general. Ubica los hechos singulares en pautas generales, y los enunciados particulares en esquemas amplios.

- Es legal. Busca leyes (de la naturaleza y de la cultura) y las aplica.

- Es predictivo. Trasciende los hechos, la experiencia, imaginando cómo pudo haber sido el pasado y cómo podrá ser el futuro. (Sánchez, Reyes, y Mejía, 2018, pp. 36-37)

Pero, este desarrollo de la investigación científica nace desde el mismo momento que nace la humanidad, donde la investigación empírica se transforma debido a la curiosidad y necesidad de los seres humanos. Esto es lo que se denomina la revolución científica que fue partiendo en diversas etapas de gran significado en el avance del hombre. Estas se consideran en cuatro grandes revoluciones. La primera consistió en la dominación de los animales y demás elementos naturales, la segunda se caracteriza por la conversión de la fuerza de la naturaleza en fuerza mecánica y la tercera se fundamenta en la capacidad de manipular las fuerzas atómicas de la materia (Guadarrama Sistos, 1988).

La última revolución tiene relación con la tercera y es la generación de tecnologías de información y comunicación que se vive en la actualidad. Es por ello, que su importancia radica debido a que investigaciones recientes han demostrado que el desarrollo tecnológico está tan influenciado por el contexto social como éste, a su vez, lo está por las nuevas tecnologías (Almiron, 2003). Es así que el impacto de esta revolución científica-técnica puede afectar el trabajo y el empleo, como se indica en la Tabla 2. 
Tabla 2. Impacto en el trabajo y empleo por parte de la revolución científica-técnica.

En primer lugar, las actuales tecnologías de punta producen un salto cualitativo, en términos de eficiencia, productividad, velocidad y calidad de los procesos, que significan un hito irreversible en los esquemas de producción y en los servicios, estableciendo el imperativo de incorporarlas como elementos esenciales para el desarrollo económico y social.

No obstante, las formas específicas de la reconversión tecnológica y las modalidades de inserción de los trabajadores varían sensiblemente en función de las concepciones y los valores políticos y culturales predominantes en cada país, dando lugar al diseño de diferentes modelos de sociedad y Estado:

Las nuevas tecnologías tienden a cerrar la brecha entre trabajo manual e intelectual, que caracterizara a los anteriores modos históricos de producción.

En el campo de la industria, los trabajadores ya no son aquellos de overol que genialmente retratara Chaplin en Tiempos modernos, sino técnicos que manejan o controlan instrumentos automatizados como robots, computadoras, sistemas de diseño o bancos de datos, lo cual requiere una calificación de nuevo tipo, que vuelve anacrónicas las tradicionales habilidades de los obreros especializados.

Los sistemas automatizados una capacitación polivalente, susceptible de cubrir un amplio espectro de funciones, con una sólida formación de base, que pueda ser reorientada hacia nuevas especializaciones ante la celeridad de los cambios tecnológicos. Pero también demandan ductilidad para integrarse en el trabajo grupal y disposición a cooperar y alimentar la solidaridad del equipo; donde el personalismo, la competencia individualista o el afán de destacarse en detrimento de los otros se vuelven cada vez más disfuncionales.

Tal vez el impacto de mayor contundencia de la revolución tecnológica en curso es la decisiva disminución en los requerimientos de tiempo de trabajo y de la participación del factor humano en la composición orgánica del capital.

Se calcula que en los más diversos ámbitos -industria, administración, servicios generales, servicios financieros, medios de comunicación e información, minería, sector agropecuario, etc.- es posible desarrollar normalmente las tareas con un promedio de tiempo de trabajo necesario inferior en un 75 por ciento al que demandaba la etapa madura de la Revolución Industrial hacia fines de los años setenta.

Fuente: (Argumedo, 1994)

En consecuencia, cada una de estas revoluciones científicas está impregnada de paradigmas científicos los cuales surgen uno después del otro, con la finalidad de refutar los anterior y permitiendo formar una cadena que describe el desarrollo del conocimiento. Es aquí que Tomas Kuhn (1962) trajo el término de inconmensurabilidad.

Es una relación que se predica de paradigmas sucesivos, alternativos o rivales, donde un paradigma es reemplazado por otro nuevo e incompatible con el anterior, sin embargo, la relación existente entre los paradigmas sucesivos (alternativos o rivales) separados por un episodio de revolución científica es no sólo de novedad o incompatibilidad, sino que queda mejor caracterizada como de inconmensurabilidad, debida a que, en el paso de un paradigma al otro, se producen cambios en: 1) el significado de los términos y conceptos científicos (entendiendo esto tanto extensional como intencionalmente, e indicando diferentes cosas sobre la población del universo y sobre el comportamiento de esa población); y, debido a ello, 2) 
cambios en (la forma (Gestalt) de ver) el mundo de investigación; y 3) en los métodos, campo de problemas y normas de solución aceptadas. (Lorenzano, 2008, pp. 242-243).

Este concepto de inconmensurabilidad no puede desviarse con otros términos que también tienen significado en la investigación científica como pueden ser la incompatibilidad o incomparabilidad lógica. Los lenguajes inconmensurables pueden ser comparados $\mathrm{y}$ evaluados racionalmente de múltiples formas, por lo que se requiere el cultivo de la imaginación y la sensibilidad hermenéutica (Bernstein, 1991). La noción de inconmensurabilidad es un aspecto ineliminable del cambio científico porque sirve a una mayor especialización y ésta es un prerrequisito para la mejor solución de enigmas, por lo que es una condición del progreso científico (Garma, 2005).

Según Kuhn, un cambio de paradigma solo es posible con el inicio de una etapa de transición, porque la ciencia normal nunca provoca ni permite un cambio, el desarrollo científico no se da por acumulación, sino por transformación o reconstrucción de un campo (Santibáñez Yáñez, 2008). Es en este sentido, que la ciencia tiende a ser acumulativa por lo que existe progreso en los conocimientos que ha generado. Esto confirma que con el paso del tiempo se ha perfeccionado el dominio por la naturaleza por lo que su desarrollo ha contribuido a una mayor eficacia en el logro de sus intereses (Boix, 2004).

Ahora, todas estas revoluciones $y$ paradigmas han permitido el crecimiento de la ciencia a medida que nace del desarrollo y las necesidades de la humanidad. Definitivamente, se debe considerar a la ciencia como el resultado de un constante proceso de evolución de conocimientos, que enriquece la historia del ser humano y lo ubica en las posiciones evolutivas de un inquebrantable avance de perfeccionamiento (Rueda de Aranguren, 2016 ). Es así que en la Tabla 3 se puede destacar las características que tipifican a la ciencia y que tiende que la investigación sea más humana.

Tabla 3. Características que tipifican a la ciencia.

Racionalidad

Objetividad

Generalidad

Sistematización
Se basa en la razón y esta constituida por conceptos y proposiciones, combinados y ordenados de acuerdo con reglas y normas lógicas.

Permite la repetición de pruebas que, mediante la observación y experimentación, facilitan la verificación de los datos obtenidos por otros investigadores.

Enuncia conceptos, agrupa y clasifica hechos particulares, busca sus cualidades esenciales y establece sus relaciones constantes con el finde generalizarlas a poblaciones mayores.

Se sustenta en ideas interconectadas y lógicas, fundamentadas en el orden y la coherencia. 


\begin{abstract}
Análisis
Constituye una herramienta para construir síntesis teóricas, tanto de los problemas como de las cosas. La investigación científica descompone un todo en pares, con el fin de descubrir su mecanismo interno, responsable de los fenómenos observados.
Claridady Parte de la formulación sencilla de problemas y su dificultad consiste en precisión identificar las causas.

Carácter acumulativo

Verificabilidad

Propone nuevos conocimientos a partir de la revisión y aplicación de los ya existentes. Si se considera que una teoría es inadecuada, el estudio científico permite presentar pruebas empíricas para remplazarla por otra nueva, ajustada a la realidad.

Ofrece conocimientos susceptibles de comprobación y de constatación con la realidad.

Empiricidad Deriva de la experiencia y de la observación de hechos, de aquello que es perceptible a los sentidos.

Veracidad Hace posible la expresión de resultados con franqueza y apego a la exactitud; no admite el engaño, la falsedad intencionada. $Y$, aparte de ser una característica, la vocación irrenunciable por la verdad es el requisito previo de la formación científica. La verdad científica no es absoluta, es relativa y fáctica.
\end{abstract}

Fuente: (Palella, y Martins, 2010)

Por lo tanto, esta investigación tiene como objetivo general analizar las teorías científicas según los postulados de Popper y Kuhn sobre investigaciones científicas. La metodología aplicada se basó en un diseño bibliográfico de tipo documental.

\section{METODOLOGÍA}

La metodología aplicada se basó en un diseño bibliográfico de tipo documental. El diseño se fundamenta en la revisión sistemática, rigurosa y profunda de material documental, donde se efectúa un proceso de abstracción científica, generalizando sobre la base de lo fundamental, partiendo de forma ordenada $y$ con objetivos precisos (Palella, y Martins, 2010).

La investigación documental se concreta exclusivamente en la recopilación de información de diversas fuentes, con el objeto de organizarla describirla e interpretarla de acuerdo con ciertos procedimientos que garanticen confiabilidad y objetividad en la presentación de los resultados (Palella Stracuzzi y Martins Pestana, 2010). Para lograr este propósito se utilizó herramientas como textos, documentos y artículos científicos publicados disponibles en la web.

\section{DESARROLLO Y DISCUSIÓN}

\section{Teorías de KR Popper}

Para el desarrollo de las teorías de Popper es necesario comprender los dos manuscritos del autor localizados en sus obras La lógica de la Investigación Científica de 1959 y Conjeturas y Refutaciones de 1969. De la primera, se tiene que los problemas epistemológicos tradicionales más importantes —los que 
guardan relación con el aumento de los conocimientos-- trascienden los dos métodos usuales de análisis lingüístico, y exigen un análisis del conocimiento científico (Popper, La lógica de la investigación científica, 1962). Sobre el análisis del conocimiento científico se deslumbra en la segunda obra.

El conocimiento, especialmente el conocimiento científico, progresa a través de anticipaciones injustificadas (e injustificables), de presunciones, de soluciones tentativas para nuestros problemas, de conjeturas. Estas conjeturas son controladas por la crítica; esto es, por intentos de refutaciones, entre las que se cuentan tests severamente críticos. Ellas pueden sobrevivir a estos tests, pero nunca pueden ser justificadas categóricamente: no se las puede establecer como indudablemente verdaderas, ni siquiera como "probables" (en el sentido del cálculo de probabilidades). La crítica de nuestras conjeturas es de importancia decisiva: al poner de manifiesto nuestros errores, nos hace comprender las dificultades del problema que estamos tratando de resolver. Es así como llegamos a adquirir un conocimiento más profundo de nuestro problema y a estar en condiciones de proponer soluciones más maduras: la misma refutación de una teoría - es decir, de una solución tentativa seria para nuestro problema- es siempre un paso adelante que nos acerca a la verdad. $Y$ es ésta la manera por la cual podemos aprender de nuestros errores. (Popper, Conjeturas y refutaciones, 1983, p. 13).

Una de las teorías más fuertes, y por la cuales le permitía cierto distanciamiento del circulo de Viena, era la teoría de la verificación, la cual al principio de sus escritos se trataba de los principios de validación y los criterios de demarcación. Según este principio, un enunciado es significativo si potencialmente existe algún medio para probarlo, confirmarlo, o con mayor probabilidad, para falsarlo e invalidarlo si se observa o produce un único ejemplo disconforme con el enunciado universal (Solé, 1994). En este sentido, la teoría de la verificación se basa en la hipótesis la cual no se demuestra lógicamente, pero si puede refutarse de forma empírica.

Existen dos ciencias en las cuales ha trabajado Popper, tanto la natural como la social, siendo la primera la más elaborada y la segunda dejando muchos cabos sueltos que no han permitido corroborar sus teorías.

Una teoría, según Popper, se mantendrá en pie hasta que sea refutada, pero no podrá demostrarse nunca que sea verdadera, ni, incluso, probable. Las teorías se fundamentarán en conjeturas y se formularán de forma provisional. Para refutar una teoría se deriva a la evidencia empírica, aunque esta por sí sola no pueda dar pruebas inequívocas, puesto que la misma percepción de los hechos no es contrastable entre sujetos observantes. El científico investigador decidirá qui conjunto de observaciones y experimentos realizará racionalmente para poder llegar a la refutación de su teoría. Estas decisiones racionales se tomarán de acuerdo con criterios externos del propio investigador y existentes en el seno de una comunidad científica, de acuerdo con el sistema de valores vigente. (Solé, 1994, p. 146). 
Muchos filósofos y científicos que han estudiado los postulados de Popper han determinado que su filosofía es de carácter deductivo, por lo que la creencia de que la probabilidad previa de cualquier ley debe ser cero. De esto, argumenta que sin importar que usualmente se encuentren cisnes blancos, el enunciado universal "todos los cisnes son blancos" nunca puede ser confirmado como verdadero (como habían creído los positivistas lógicos) puesto que en el futuro puede haber un cisne negro (Redman, 1995). Por lo que también, sostiene que la ciencia y la vida misma no comienzan con el registro pasivo de lo que ocurre en la realidad sino con la formulación de teorías para solucionar los problemas que individuos tienen que enfrentar conforme a los fines que persiguen (Olivares, 2006). Esto es a lo que se refiere al método crítico o al de ensayo y error. Según Popper (1974) plantea:

$$
\mathrm{P}_{1} \rightarrow \mathrm{TT} \rightarrow \mathrm{EE} \rightarrow \mathrm{P}_{2}
$$

...todas las discusiones científicas comienzan con un problema $\left(\mathrm{P}_{1}\right)$, para el cual ofrecernos una especie de solución tentativa -una teoría tentativa (TT); esta teoría luego es criticada, en un esfuerzo por eliminar el error (EE) y, corno en el caso de la dialéctica, este proceso se renueva a sí mismo: la teoría y su revisión crítica dan lugar a nuevos problemas $\left(\mathrm{P}_{2}\right)$. (Redman, 1995, pág. 121).

Nuevamente, esta teoría es fundamental para el desarrollo del conocimiento científico lo cual refleja tres pasos importantes: la contrastación, la falsación y la corroboración. La contrastación es el análisis crítico, la coherencia con otras teorías y la confrontación con la realidad; la falsabilidad se refiere a la progresiva aproximación a teorías verdaderas mediante tentativas de refutación; y la corroboración es la confirmación temporal de las teorías (Burgos, 2011). Por lo cual, la clásica sabiduría de la duda, el paréntesis se ha convertido de la mano de Popper en un paradójico empujón para actuar, perfecto salvoconducto para tomar decisiones que dejarán de ser evidentes mañana (Miguel García, 2000).

En este sentido, las teorías más resaltantes de Karl Popper se pueden distinguir en la Tabla 4, las cuales plantean el núcleo epistemológico de la ciencia.

Tabla 4. El núcleo epistemológico según Karl Popper.

\section{CRÍTERIOS}

Inducción como método científico.

\section{CARACTERÍSTICAS}

No se debe considerar como método científico la inducción y cuál sería el procedimiento adecuado para hacer ciencia. Ahora bien, se debe diferenciar el método de la inducción y la inducción como problema. El método de la inducción consiste en obtener afirmaciones generales partiendo de enunciados particulares o individuales. Esto, desde la perspectiva lógica, discrepa considerablemente de la verdad que se quiere alcanzar, pues en algún momento la afirmación resultará ser falsa, debido, a que por más numerosos que sean los ejemplos, nunca abarcará la totalidad de las experiencias. 


\section{CRÍTERIOS CARACTERÍSTICAS}

Criterio de demarcación y falsabilidad

La experiencia como método

Objetividad científica
El criterio de demarcación propuesto por los inductivistas exige que todo enunciado de la ciencia empírica sea susceptible de una decisión definitiva con respecto a su verdad o falsedad.

Sí es completamente inadmisible el método inductivo, también lo es su criterio de demarcación, es decir, la verificación. Se ha señalado que lógicamente no se pueden deducir teorías partiendo de enunciados particulares; o, lo que es lo mismo, de lo particular no podemos sacar lo universal. La verificación empírica tampoco es posible, ya que no podemos contar con la experiencia total de los casos particulares. En tales condiciones, un sistema científico no debe pretender ser seleccionado para siempre, sino, ser seleccionado, después de múltiples contrastaciones, provisionalmente, mientras no sea refutado. Así se prueba una de las características fundamentales de la ciencia: el fomento de la crítica y la discusión racional.

Popper propone un nuevo criterio de demarcación, esto es, uno que pueda separar realmente las ciencias empíricas, de las seudociencias: la falsabilidad.

Es decir, se recurre a la experiencia, no para buscar en ella ejemplos que reafirmen una teoría, sino que, por el contrario, para encontrar ejemplos que falsen tal teoría. De este modo se encuentra con que: muchos ejemplos (casos particulares) no logran reafirmar una teoría, pero basta un solo ejemplo que contradiga la teoría para que ésta sea falsa.

La falsabilidad es, seguramente, la mayor contribución de Popper a la Epistemología. La ciencia se construye con base en la razón y la experiencia; no se trata de buscar hechos que confirmen las teorías, sino hechos que las refuten.

La experiencia es el método mediante el cual el sistema de la ciencia empírica se diferencia de otros sistemas teóricos cuya estructura lógica sea parecida al sistema aceptado en un determinado momento. Popper considera que hablar de ciencia empírica es una tarea difícil pero que es necesario entender la experiencia como el método por el cual las teorías se refieren al mundo real, es decir, se someten a las contrastaciones y las resisten.

Popper, a diferencia de los positivistas considera, que la teoría precede a la experiencia. La experiencia sirve como medio para falsar las teorías, por esto es indispensable que los enunciados de la ciencia empírica sean falsables y, por lo tanto, aceptables universalmente. De este modo la falsabilidad, abre el camino a la objetividad.

La objetividad es una característica propia de la ciencia. La ciencia es un conjunto de argumentos lógicamente válidos, y como tal, debe ser un sistema de enunciados contrastables.

La objetividad de los enunciados depende de su capacidad para ser contrastados; es decir, de ser expuestos a la falsabilidad.

Los enunciados científicos son enunciados contrastables intersubjetivamente $y$, por lo mismo, tal contrastación puede efectuarla cualquiera sin más condición que la de que se realice de manera apropiada. 


\section{CRÍTERIOS}

Contrastación

deductiva de teorías

Carácter del
método científico

\section{CARACTERÍSTICAS}

La contrastación deductiva, es el método de criticar y escoger teorías, es una reconstrucción racional. Consiste en presentar, a título provisional, un enunciado, a partir del cual se pueden sacar conclusiones teniendo en cuenta las relaciones lógicas que existen entre ellas.

Es importante destacar que la contrastación, basada en la falsabilidad, liga la experiencia a la inferencia lógica. No se trata de una inferencia generalizadora positiva sino negativamente: de la falsedad de los enunciados observacionales podemos inferir la falsedad de los enunciados universales, vale decir, de las teorías.

La epistemología o la lógica de la investigación científica vienen a identificarse con la teoría del método científico, es decir, con el modo correcto de proceder en el campo de la ciencia. Esto significa que la teoría del método científico tiene que ver con las decisiones acerca del modo de haberla con los enunciados científicos. Popper admite la necesidad de un análisis puramente lógico de las teorías, pero considera que jamás se puede presentar una refutación concluyente ya que, siempre se puede decir que los resultados experimentales no son dignos de confianza, que las discrepancias son aparentes o que desaparecerán en la medida en que se comprenden los hechos.

Fuente: (Burgos, et.al., 2007)

Ahora, estas teorías han sido poco probables de demostrar en las ciencias sociales, sobre todo con los avances científicos ocurrido en este último siglo. Por lo que, el desarrollo tecnológico y científico era directamente proporcional a las políticas aplicadas en los estados, es decir a la democracia impartida en dicha nación. Así que:

Hipótesis 1: progreso científico e industrial depende en gran medida de la democracia.

Crítica: A lo largo de la historia se ha producido un notable progreso tecnológico, incluso en épocas y lugares con muy poca libertad de pensamiento. La hipótesis hay que reformularla en el sentido de que el progreso científico y económico ha sido mayor donde ha existido una mayor libertad (hipótesis 2).

Falsedad: rechazaría la nueva hipótesis si una dictadura de cualquier tipo (comunista, fascista u otra) logra en términos relativos un progreso científico superior al de un país democrático comparable. (Moliní, 1999, p. 3).

La hipótesis 2 es completamente refutable cuando en la década de los 50's la Unión Soviética tenía el impulso tecnológico a la par de los Estados Unidos tras la carrera espacial, cuando fue la primera nación en tener un hombre orbitando en el espacio. Por lo que, una posibilidad es que se cometió el error de no incluir en su formulación la variable tiempo, asimismo, podría ser que la hipótesis fuese rechazable en la sociedad industrial, pero que sin embargo adquiere validez en la sociedad del conocimiento (Moliní, 1999).

\section{Teorías de Tomas Kuhn}

Existes dos obras de Tomas Kuhn que se van a tomar en cuenta en esta investigación, una 
es la estructura de la revolución científica (1962) y el otro es el camino hacia la estructura (2000). Una parte importante en los estudios de Kuhn es la importancia que tiene la experiencia o valores de una persona para poder agregar un significado a un concepto o teoría. Los valores son absorbidos a través de la educación de los miembros de una comunidad, pero ello no lo legitima, son adaptados más por presión social que por elección fundada en razones (Garma, 2005).

Es esta una de las razones por las que las teorías de Kuhn se han movido dentro de las limitaciones de conocimientos epistemológicos y de psicología del conocimiento. Existe una convicción de que Kuhn ha querido resaltar siempre ha sido el producto grupal y el hecho histórico, por lo que la naturalización del conocimiento científico no pasaría más que por el estudio socio-histórico de las condiciones en que se produce (Brunetti, 2010). Es así que la vía que analiza la ciencia es el enfoque histórico, dedicándose en primera instancia al estudio de la historia de la ciencia y revisando, así como surgen diversas cuestiones que muestran un contraste entre dos concepciones de la misma (Rueda de Aranguren, 2016 ). Según SánchezCerezo (2003) las dos concesiones son:

Por un lado, la ciencia entendida como una actividad completamente racional y controlada; tal como la presenta el Círculo de Viena (19221936), que, para la concepción científica del mundo, se ocupaba principalmente de la lógica de la ciencia, fundamentando la filosofía como una disciplina encargada de distinguir entre lo que es ciencia y lo que no es, y de la elaboración de un lenguaje común a todas las ciencias. Y, por otro lado, la ciencia entendida como una actividad concreta que se ha venido dando a lo largo de los siglos y que en cada época histórica presenta peculiaridades y características propias. (citado por Rueda de Aranguren, 2016, p. 3).

A través de indagar en la historia de la ciencia presenta dos términos que se convierten en el fuerte de sus teorías, uno es el paradigma y el otro la comunidad científica. Es así que su punto de vista se encuentra en un lado por estos dos términos y por el otro están el de "ciencia normal" y de "ciencia de crisis", donde la transición de estos tipos de ciencia es el proceso caracterizado como "revolución científica" (Krotz, 1981).

De estos términos el que más ha generado controversia, desde la publicación de la estructura de la revolución científica, ha sido paradigma. Esto ha permitido que mucho de sus detractores tomen reservas y malentendidos. Para ello, el mismo Kuhn años más tardes busca la forma de poder establecer un criterio único y veraz de este término. Señala Kuhn (2007) que el término se usa con dos sentidos diferentes:

Por un lado, hace alusión a toda la constelación de creencias, valores, técnicas y demás, compartidos por los miembros de una comunidad dada; y por otro denota las soluciones concretas a rompecabezas que, usadas como modelos o ejemplos pueden sustituir a las reglas como base para la solución de los restantes rompecabezas de la ciencia normal. (citado por Briceño, 2009, p. 7-8).

Para poder dilucidar entre los paradigmas presentes en diferentes teorías o postulados es necesario aplicar el razonamiento científico. En la historia de la ciencia, las controversias científicas y entre paradigmas se desarrollan principalmente a través de razonamiento 
científico, por lo que es el principal medio racional para establecer desacuerdos o alcanzar acuerdos y de interrelación entre paradigmas diferentes (Guillaumin, 2009).

Tras el debate del significado de paradigma, también nació el término inconmensurabilidad que se relaciona con esa interrelación entre paradigmas diferentes.

$\mathrm{Su}$ propósito principal era el de indicar las formas en las que los paradigmas-teorías podían y no podían ser comparados; además la incompatibilidad no debe confundirse o ser asimilada con la inconmensurabilidad, ya que la incompatibilidad es un concepto lógico que presupone -como hace notar el propio Kuhn- un lenguaje común en el que podemos especificar relaciones lógicas incompatibles. (Bernstein, 1991, p. 8).

Es aquí donde los grandes filósofos han tenido gran controversia con sus postulados, más al contrario los investigadores de las ciencias sociales, en especial, los de psicología y comportamiento del individuo en la búsqueda del conocimiento. De los primeros Kuhn no desarrolla un juicio histórico del concepto mismo de paradigma, a pesar de que su perspectiva es histórica; por otra, no considera a cabalidad las teorías de filosofía para explicar los conceptos de inconmensurabilidad y regla (Santibáñez Yáñez, 2008).

Muchos científicos en el área comentan que dicho postulado salió cuando apenas era recién graduado de la universidad y que por ende en el transcurrir de su vida fue cambiando cada uno de esto gracias a la experiencia y a su análisis profundo. En este sentido, Kuhn permaneció, bajo este término de inconmensurabilidad, en una filosofía de lenguaje por lo que tiende a ser visto desde la óptica de la hermenéutica para que dicho conflicto mitigue (Verdejo, 2019). En cambio, se convertía en una referencia de primer orden para sociólogos, antropólogos y demás científicos sociales., donde la filosofía kuhniana encontraba un sentido de autopercepción disciplinar más sustantivo $\mathrm{y}$ más alentador que el brindado por el racionalismo crítico (Melogno, 2020).

Es por ello, necesario poder establecer el desarrollo de sus teorías a través de su misma historia y poder así comprender su proceso evolutivo en las filosofías, pero sobre todo en las ciencias sociales, específicamente en las teorías cognitivas, tal como se describe en el Tabla 5. 
Tabla 5. Etapas en el desarrollo de las teorías de Tomas Kuhn.

\begin{tabular}{|c|c|}
\hline ETAPAS & CARACTERÍSTICAS \\
\hline $\begin{array}{l}\text { El primer Kuhn. La } \\
\text { relación temprana } \\
\text { entre Kuhn y la } \\
\text { psicología del } \\
\text { conocimiento }\end{array}$ & $\begin{array}{l}\text { El esquema conceptual cumple con fines psicológicos y lógico-cognitivos. Pero } \\
\text { aquí Kuhn ve, en los aspectos psicológicos, algo que sólo tiene que ver con la } \\
\text { tranquilidad que crea en la mentalidad del científico la confianza en un } \\
\text { determinado esquema conceptual con el cual poder trabajar. Esta sensación la } \\
\text { aporta el hecho de que los científicos descansan en una serie de creencias } \\
\text { admitidas sin discusión. No está, por lo tanto, apelando a una psicología del } \\
\text { conocimiento sino a una psicología de la afectividad o de estados anímicos. } \\
\text { Pero los aspectos lógicos que menciona son fundamentalmente cognitivos. De } \\
\text { manera que correspondería incorporar estos últimos dentro de una psicología } \\
\text { del conocimiento, como lo hará luego. } \\
\text { Los paradigmas ejercen sobre la mente de los científicos una influencia } \\
\text { implícita que les hace aceptar algunas cuestiones básicas dándolas por } \\
\text { sobreentendidas. Su formación y la tradición de la investigación que comparten } \\
\text { proponen la coherencia necesaria para evitar cuestionamientos radicales. } \\
\text { Los casos que cita Kuhn son netamente cognitivos. Se trata de que la } \\
\text { experiencia de ciertos aspectos del mundo conocido actúa condicionando las } \\
\text { cogniciones siguientes. } \\
\text { El panorama de la ciencia, tal como ha sido planteado por Kuhn, avala una } \\
\text { concepción del desarrollo científico como un proceso discontinuo y no } \\
\text { acumulativo. }\end{array}$ \\
\hline $\begin{array}{l}\text { La etapa de } \\
\text { transición. Los } \\
\text { ejemplares como } \\
\text { construcciones } \\
\text { psicológicas }\end{array}$ & $\begin{array}{l}\text { Cuando Kuhn decide reformular su primera concepción amplia de paradigma } \\
\text { por una más restringida de ejemplares incluye notas que son netamente } \\
\text { psicológicas. Los paradigmas, en tanto capacidad para resolver problemas, } \\
\text { residen como saber práctico en el psiquismo de los científicos. } \\
\text { El aprendizaje se da por medio de mostraciones repetidas (repetición), } \\
\text { corrección y fijación. Ahora bien, según la teoría del refuerzo, los aprendizajes } \\
\text { tienden a fijarse en la medida que produce algún tipo de satisfacción en el } \\
\text { individuo, mientras que cuando su efecto es insatisfactorio, o actúa a modo de } \\
\text { castigo o error verificado, se tiende a eliminar la conducta. } \\
\text { Kuhn eligió una psicología de tinte asociacionista porque responde a sus } \\
\text { motivaciones personales o al conocimiento sobre la psicología del } \\
\text { conocimiento de que disponía entonces. La etapa de transición no lo es sólo } \\
\text { desde el punto de vista epistemológico, sino también desde la elección de la } \\
\text { teoría psicológica del conocimiento. Lo cierto es que resulta particularmente } \\
\text { interesante verificar que cuando Kuhn necesita responder a sus críticos } \\
\text { bosqueje una justificación desde procesos psicológicos. Si leyéramos el telón de } \\
\text { fondo de la psicología que sustenta en esta etapa la podríamos caracterizar } \\
\text { como empirista, asociacionista y hasta conductista }\end{array}$ \\
\hline
\end{tabular}


El último Kuhn. El problema del cambio científico
Para la misma época Kuhn manifiesta inclinarse por la investigación de cómo ocurren los cambios científicos. Sobre este respecto comienza a entrever que las estructuras científicas deben cambiar, aunque no de una manera tan radical como lo suponía en La estructura de la revolución científica.

Kuhn propone que el cambio científico puede incluir yuxtaposiciones de elementos de un núcleo tradicional con otros sacados de expansiones de ese mismo núcleo.

La adopción de un marco cognitivista en el último Kuhn queda justificada por razones que su propia epistemología requería. La psicología no puede eludir la diferenciación entre dos tipos de procesos cognitivos presentes en el modelo epistemológico de Kuhn. Uno es el proceso de adquisición de conceptos vinculados con el paradigma vigente; otro es el que genera un cambio conceptual y por lo tanto representa, en el modelo epistemológico kuhniano, una transición revolucionaria. Los dos procesos forman parte de la investigación en psicología cognitiva.

Fuente: (Brunetti, Thomas Kuhn:¿ epistemólogo o psicólogo de la ciencia?, 2013)

Sin embargo, es imposible descartar su influencia en el desarrollo del progreso científico por medio de las investigaciones científicas.

El progreso científico, para Kuhn, es un proceso de desarrollo ateleológico, un proceso cuyas etapas sucesivas se caracterizan solamente por alcanzar una comprensión cada vez más detallada y refinada de la naturaleza. Esta definición que, por una parte, es manifestación de la connotación social que Kuhn da a la actividad científica, muestra, también, el talante pragmático de la propuesta kuhniana. En efecto, este autor parece considerar que es más eficaz estudiar el progreso de la ciencia en función del incremento de la articulación y la especialización de las construcciones teóricas, es decir de su utilidad práctica, que analizar su relación con la existencia de una explicación objetiva, plena y verdadera de la naturaleza. (Campos, 2003, p. 279).

\section{Relación entre Popper y Kuhn}

El desarrollo de la ciencia tiene dos perspectivas diferentes según estos grandes científicos. Popper recomienda que el científico, independientemente de que sea teórico o experimental, propone enunciados, hipótesis o sistemas de teorías y los contrasta paso a paso por medio de observaciones y experimentos; y Kuhn sostiene que toda actividad científica se desarrolla bajo un paradigma (García Jiménez, 2008).

A través de los años ha existido debate entre estos grandes pensadores los cuales cada uno refuto sus postulados, proponiendo mejoras en los mismos. Kuhn tiene su diferencia respecto a la tesis de la falsación porque no toma en consideración la ciencia normal; en cambio Popper comenta que este término es impreciso, sino que, además, propone una alternativa que centra su argumento en la educación del científico (Jaramillo y Aguirre, 2004). Popper dice que ningún científico hace ciencia normal, así como 
también insiste que un paradigma es más bien un programa de investigación que una teoría dominante y que la lógica de Kuhn es de Relativismo Histórico (Sequeiros, 2001). Por otra parte, Kuhn estimaba que Popper "era demasiado positivista" para su modo de considerar la ciencia y que consideraba que las teorías posteriores incluyen acumulativamente a las anteriores; y Kuhn piensa que las ciencias no avanzaban por un carácter acumulativo (Daros, 2007).

A pesar de estas críticas Kuhn tendió a poder converger ambas teorías, sin ánimos de buscar controversias. En sus discursos propone estar más cerca de los postulados de Popper que de los de psicologista como se le acusaba, aunque si se diferenciaba de algunas teorías planteadas por Karl. Kuhn reconocía que sus puntos de vista y los de Popper, eran "muy aproximadamente idénticos" y que "ambos rechazaban el punto de vista de que la ciencia progresa por acumulación (Daros, 2007). Es decir, la ciencia progresa en el suscitar nuevos problemas (Barreto, 2014).

En consecuencia, la propuesta de Popper tiene carácter racional, mientras que la de Kuhn tiene carácter irracional o de psicología de masas (Rosales López, 2005). La actitud de Popper permite divorciar a la epistemología de cualquier clase de consideraciones psicológicas y/o sociológicas acerca de la ciencia, pero para Kuhn estas últimas resultan insoslayables en el examen no solo del desarrollo de la ciencia, sino también del método científico (Barreto, 2014).

\section{REFERENCIAS}

Almiron, N. (2003). Sobre el progreso en una era de revolución científico-tecnológicodigital. Ámbitos. Revista Internacional de Comunicación, (10), 1-10. Obtenido de https://www.redalyc.org/pdf/168/16801 029.pdf
Alzugaray, S., Mederos, L., \& Sutz, J. (2011). La investigación científica contribuyendo a la inclusión social. Revista Iberoamericana de Ciencia, Tecnología y Sociedad-CTS, 6(17), 11-30. Obtenido de https://www.redalyc.org/pdf/924/92422 634001.pdf

Argumedo, A. (1994). Impacto de la Revolución Científico-Técnica. El imperio del conocimiento. Revista" Encrucijadas, (4). Obtenido de https://www.bibliopsi.org/docs/CBC/SOC IEDAD \%20Y\%20ESTAD0/06/AlciraArgu medo.pdf

Barreto, C. L. (2014). Falsabilidad y revoluciones científicas. Karl Popper y Thomas Kuhn. Tradición, segunda época, (14), 84-96. Obtenido de http://168.121.49.88/index.php/Tradicion /article/view/340

Bernstein, R. J. (1991). Una revisión de las conexiones entre inconmensurabilidad y otredad. Isegoría, (3), 5-25. Obtenido de http://isegoria.revistas.csic.es/index.php/i segoria/article/view/364

Boix, I. J. (2004). Pluralismo epistemológico: Entre el sueño objetivista y la inconmensurabilidad de paradigmas. Utopía y praxis latinoamericana: revista internacional de filosofía iberoamericana $y$ teoría social, (24), 51-65. Obtenido de https://dialnet.unirioja.es/servlet/articulo ?codigo $=2692983$

Briceño, T. (2009). El paradigma científico y su fundamento en la obra de Thomas Kuhn. Tiempo y Espacio, 19(52), 285-297. Obtenido de https://dialnet.unirioja.es/servlet/articulo ?codigo $=3579139$

Brunetti, J. (2010). Algunas consideraciones sobre la relación entre epistemología y psicología en Thomas Kuhn. BAJO PALABRA. Revista de Filosofía, II Época, № 5, 419-428. Obtenido de https://repositorio.uam.es/bitstream/han dle/10486/6141/37342_34.pdf?sequence $=1$ 
Brunetti, J. (2013). Thomas Kuhn: $i$ epistemólogo o psicólogo de la ciencia? Revista Iberoamericana de Ciencia, Tecnología y Sociedad-CTS, 8(22), 191-212. Obtenido de https://www.redalyc.org/pdf/924/92425 714010.pdf

Bunge, M. (2003). Información+ evaluación= conocimiento. Pliegos de Yuste, 1, 75-84.

Burgos, C. (2011). Hacia una teoría educativa desde el pensamiento de Karl Popper. Barcelona, España: In XII Congreso Internacional de Teoría de la Educación, Universidad de Barcelona. Obtenido de https://www.academia.edu/download/38 777513/Popper_K_OK.pdf

Burgos, C. E., Retamoso, G., Agudelo, J. C., \& Murgueitio, M. A. (2007). Karl R. Popper. Génesis de una teoría Educativa. Civilizar. Ciencias Sociales y Humanas, 7(12), 151$169 . \quad$ Obtenido de https://www.redalyc.org/pdf/1002/1002 20305011.pdf

Campos, M. S. (2003). La relación teoríaexperiencia en la epistemología de Thomas S. Kuhn. Roma, Italia: Tesis para optar al título de Dcotor en Filosofía de la Pontificia Universitas Sanctae Crucis, Facultas Philosophiae. Obtenido de https://www.unav.es/gep/TesisDoctorale s/TesisSanchezCampos.pdf

Daros, W. (2007). Los condicionamientos sociales en los paradigmas científicos: Popper y Kuhn. Invenio, 10(18), 47-74. Obtenido de https://www.redalyc.org/pdf/877/87701 805.pdf

Garcés Paz, H. (2000). Investigación científica. Quito, Ecuador: Ediciones Abya-Yala Obtenido de https://digitalrepository.unm.edu/cgi/vie wcontent.cgi?article $=1356 \&$ context=abya yala

García Jiménez, L. (2008). Aproximación epistemológica al concepto de ciencia: una propuesta básica a partir de Kuhn, Popper, Lakatos y Feyerabend. Andamios 4.8, 185202.

Obtenido http://www.scielo.org.mx/pdf/anda/v4n8 /v4n8a8.pdf

Garma, A. (2005). Thomas Kuhn y la racionalidad científica. Inconmensurabilidad y verdad. A Parte Rei Revista de Filosofía 40, 1-9. Obtenido de http://serbal.pntic.mec.es/cmunoz11/ama nda40.pdf

Guadarrama Sistos, R. (1988). La tercera revolución científico-tecnológica de la humanidad. Estudios Políticos, (1), 4-12. Obtenido de http://revistas.unam.mx/index.php/rep/a rticle/view/60084

Guillaumin, G. (2009). El relativismo epistemológico visto a través de la teoría del cambio científico de Thomas Kuhn. Relaciones. Estudios de historia y sociedad, 30(120), 139-164. Obtenido de http://www.scielo.org.mx/pdf/rz/v30n12 0/v30n120a5.pdf

Jaramillo, L., y Aguirre, J. (2004). La controversia Kuhn-Popper en torno al progreso científico y sus posibles aportes a la enseñanza de las ciencias. Cinta de Moebio. Revista de Epistemología de Ciencias Sociales, (20), 83-92. Obtenido de https://adnz.uchile.cl/index.php/CDM/arti cle/view/26111

Krotz, E. (1981). ¿Ciencia normal o revolución científica? Constructores de Otredad, 34., 34-47. Obtenido de http://www.academia.edu/download/334 39108/Una_introduccion_a_la_Antropologi a_Social_y_Cultural.pdf\#page $=33$

Lorenzano, P. (2008). Inconmensurabilidad teórica y comparabilidad empírica: el caso de la genética clásica. Análisis filosófico, 28(2), 239-279. Obtenido de https://www.redalyc.org/pdf/3400/3400 30298005.pdf

Martínez, A., y Ríos, F. (2006). Los conceptos de conocimiento, epistemología y paradigma, como base diferencial en la orientación metodológica del trabajo de grado. Cinta de Moebio. Revista de Epistemología de Ciencias Sociales, (25), 111-121 
Melogno, P. (2020). MÁS ALLÁ DE LOS PARADIGMAS: MIRADAS ACTUALES SOBRE THOMAS KUHN. Cuadernos de Filosofía, (38), 7-17. Obtenido de http://revistas.udec.cl/index.php/cuaderno s_de_filosofia/article/download/2461/274 1

Méndez, I., Namihira, D., Moreno, L., y Sosa, C. (2001). El protocolo de investigación. México DF: Trillas. Obtenido de http://www.academia.edu/download/380 62890/BUENO_2.pdf

Miguel García, F. (2000). La fascinación por Popper en la epidemiología contemporánea. Rev Esp Salud Pública; 74, 323-326. Obtenido de https://www.scielosp.org/pdf/resp/2000.v 74n4/323-326/es

Miranda Montecinos, A. (2013). Plagio y ética de la investigación científica. Revista chilena de derecho, 40(2), 711-726. Obtenido de https://scielo.conicyt.cl/pdf/rchilder/v40n 2/art16.pdf

Moliní, F. (1999). Proactividad: el método científico de Karl Popper aplicado al futuro. Encuentros multidisciplinares. Obtenido de https://repositorio.uam.es/bitstream/hand le/10486/684768/EM_3_4.pdf?sequence=1

Morone, G. (2013). Métodos y técnicas de la investigación científica. Valparaíso, Chile: Documento de trabajo. Pontificia Universidad Católica de Valparaíso. Sistema de Biblioteca. Obtenido de https://www.academia.edu/download/341 42249/metodologias_investigacion.pdf

Olivares, E. H. (2006). Observación, teorías y valores a la luz de la filosofía de Popper. CIENCIA ergo-sum, Revista Científica Multidisciplinaria de Prospectiva, 13(2), 200210.

Obtenido

de https://www.redalyc.org/pdf/104/104132 12.pdf

Palella Stracuzzi, S., y Martins Pestana, F. (2010). Metodología de la investigación cuantitativa. Caracas, Venezuela: FEDUPEL, Fondo Editorial de la Universidad Pedagógica Experimental Libertador.
Popper, K. (1962). La lógica de la investigación científica. Madrid, España: Editorial Tecnos, S. A. Obtenido de http://www.raularagon.com.ar/biblioteca/l ibros/Popper\%20Karl\%20-

\%20La\%20Logica\%20de\%20la\%20Investi gacion\%20Cientifica.pdf

Popper, K. (1983). Conjeturas y refutaciones. Barcelona, España: Ediciones Paidos. Obtenido de https://elartedepreguntar.files.wordpress.c om/2009/06/popper-karl-conjeturas-yrefutaciones.pdf

Redman, D. (1995). La teoría de la ciencia de Karl Popper y la econometría. Cuadernos de economía (Santafé de Bogotá), 14(23), 118$149 . \quad$ Obtenido de https://dialnet.unirioja.es/servlet/articulo? codigo $=4934938$

Romero, A. (2002). Las redes de información y su importancia para la investigación científica. Revista Venezolana de Gerencia, 7(19), 425-441. Obtenido de https://www.redalyc.org/pdf/290/290019 06.pdf

Rosales López, V. (2005). Breve revisión a los puntos fundamentales de los programas de investigación de TS Kuhn y Karl Popper. Revista FACES de la Universidad de Carabobo. Obtenido de http://servicio.bc.uc.edu.ve/faces/revista/a 7n15/7-15-3.pdf

Rueda de Aranguren, D. M. (2016 ). Transformación de los paradigmas en revolución científica. Perspectiva de Thomas Kuhn. Analéctica, 2(18), http://portal.amelica.org/ameli/jatsRepo/2 51/2511354004/2511354004.pdf.

Sánchez Carlessi, H., Reyes Romero, C., y Mejía Sáenz, K. (2018). Manual de términos en investigación científica, tecnológica $y$ humanística. Lima, Perú: Universidad Ricardo Palma, Vicerrectorado de Investigación. Obtenido de http://repositorio.urp.edu.pe/handle/URP/ 1480 
Santibáñez Yáñez, C. (2008). Ciencia, inconmensurabilidad y reglas: crítica a Thomas Kuhn. Revista de filosofía, 64, 163182. Obtenido de https://scielo.conicyt.cl/pdf/rfilosof/v64/ art11.pdf

Sautu, R. (1997). Acerca de qué es y no es investigación científica en ciencias sociales. La trastienda de investigación, 227-240. Obtenido de http://www.fapyd.unr.edu.ar/wpcontent/uploads/2015/09/WainermanSautu-cap-7.pdf

Sequeiros, L. (2001). Popper y Kuhn: Veinte años después. Reflexión didáctica en el Centenario (1902-2002) del nacimiento de Karl R. Popper. Enseñanza de las Ciencias de la Tierra, 9(1), 2-12. Obtenido de https://www.raco.cat/index.php/ECT/arti cle/view/88773/132848
Solé, C. (1994). Karl Popper. Papers: revista de sociologia, 145-147. Obtenido de https://www.raco.cat/index.php/Papers/a rticle/download/25257/58541

Verdejo, A. E. (2019). El giro filosófico en el pensamiento de Thomas Kuhn sobre la inconmensurabilidad de las teorías científicas. Interciencia, 44(11), 653-659. Obtenido de https://www.interciencia.net/wpcontent/uploads/2019/12/07_653_E_Espi noza_v44n11-1.pdf 